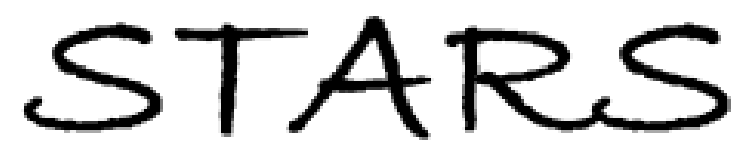

University of Central Florida

STARS

$1-1-2012$

\title{
Direct generation of intense extreme-ultraviolet supercontinuum with 35-fs, 11-mJ pulses from a femtosecond laser amplifier
}

Bin Zeng

Wei Chu

Guihua Li

Jinping Yao

Jielei $\mathrm{Ni}$

See next page for additional authors

Find similar works at: https://stars.library.ucf.edu/facultybib2010

University of Central Florida Libraries http://library.ucf.edu

This Article is brought to you for free and open access by the Faculty Bibliography at STARS. It has been accepted for inclusion in Faculty Bibliography 2010 s by an authorized administrator of STARS. For more information, please contactSTARS@ucf.edu.

\section{Recommended Citation}

Zeng, Bin; Chu, Wei; Li, Guihua; Yao, Jinping; Ni, Jielei; Zhang, Haisu; Cheng, Ya; Xu, Zhizhan; Wu, Yi; and Chang, Zenghu, "Direct generation of intense extreme-ultraviolet supercontinuum with 35-fs, 11-mJ pulses from a femtosecond laser amplifier" (2012). Faculty Bibliography 2010s. 3547.

https://stars.library.ucf.edu/facultybib2010/3547

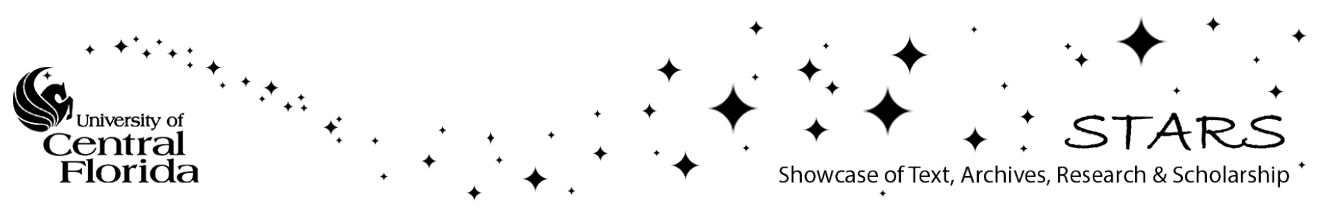


Authors

Bin Zeng, Wei Chu, Guihua Li, Jinping Yao, Jielei Ni, Haisu Zhang, Ya Cheng, Zhizhan Xu, Yi Wu, and Zenghu Chang 


\title{
Direct generation of intense extreme-ultraviolet supercontinuum with 35-fs, 11-mJ pulses from a femtosecond laser amplifier
}

\author{
Bin Zeng, ${ }^{1,2}$ Wei Chu ${ }^{1,2}$ Guihua Li ${ }^{1,2}$ Jinping Yao, ${ }^{1}$ Jielei Ni ${ }^{1,2}$ Haisu Zhang,${ }^{1,2}$ \\ Ya Cheng,,$^{1, *}$ Zhizhan $\mathrm{Xu},{ }^{1, \dagger} \mathrm{Yi} \mathrm{Wu},{ }^{3}$ and Zenghu Chang $^{3}$ \\ ${ }^{1}$ State Key Laboratory of High Field Laser Physics, Shanghai Institute of Optics and Fine Mechanics, Chinese Academy of Sciences, \\ Shanghai 201800, China \\ ${ }^{2}$ Graduate School of Chinese Academy of Sciences, Beijing 100039, China \\ ${ }^{3}$ Department of Physics, University of Central Florida, Orlando, Florida 32816, USA and CREOL, University of Central Florida, \\ Orlando, Florida 32816, USA
}

(Received 27 November 2011; published 30 March 2012)

\begin{abstract}
We report on the generation of an intense extreme-ultraviolet (EUV) supercontinuum with photon energies spanning from 35 to $50 \mathrm{eV}$ (i.e., supporting an isolated attosecond pulse with a duration of $\sim 271$ as) by loosely focusing $35 \mathrm{fs}, 11 \mathrm{~mJ}$ pulses from a femtosecond laser amplifier into a 10-mm-long gas cell filled with krypton gas. The dramatic change in spectral and temporal properties of the driver pulses after passing through the gas cell indicates that propagation effects play a significant role in promoting the generation of the EUV supercontinuum.
\end{abstract}

DOI: 10.1103/PhysRevA.85.033839

PACS number(s): 42.65.Ky

\section{INTRODUCTION}

The successful generation of attosecond pulses has enabled time-resolved investigation of light-matter interaction at the natural time scale of electrons [1]. To date, highorder harmonic generation (HHG) still remains the standard workhorse for a generation of isolated attosecond pulses (IAPs) [2], which requires the use of either externally compressed carrier-envelope-phase- (CEP) controlled few-cycle pulses [3] or wave-form-synthesized multicycle pulses [4,5]. The employment of sophisticated wave-form tailoring and control techniques often leads to increased complexity of the experimental setup, limited driver pulse energies, and extra optical losses of the driver pulses. These obstacles hamper the widespread use of attosecond technology for revealing ultrafast dynamics in atomic, molecular, and condensed-matter systems. Therefore, new techniques that can greatly simplify the attosecond pulse generation and make more efficient use of the driver pulses are highly in demand.

Recently, it has been shown that IAPs can be directly generated from a filament formed by loosely focusing the driver laser beam into a semi-infinite gas cell filled with argon at atmospheric pressure [6]. In this case, the multicycle driver pulses with a pulse energy of $\sim 1 \mathrm{~mJ}$ and an initial pulse duration of $\sim 35$ fs were automatically transformed to pulses with complex temporal structures during the filamentation process. In particular, intensity spikes with a duration approaching a single optical cycle could emerge in the transformed pulses within which IAPs are produced. Obviously, this technique can greatly simplify the attosecond pulse generation as it does not have any specific requirements on the initial wave form of driver pulses except the control of the CEP. Recently, when we carried out HHG experiments with high-energy driver pulses aimed at producing high-brightness $\mathrm{x}$-ray radiation (i.e., the original

\footnotetext{
*ya.cheng@siom.ac.cn

†zzxu@mail.shcnc.ac.cn
}

purpose of this experiment was to generate intense highorder harmonics but not supercontinuum), surprisingly, we observed that the extreme-ultraviolet (EUV) supercontinuum emerges when Fourier-transform-limited $\sim 35$-fs, 11 -mJ femtosecond laser pulses were loosely focused into a 10-mm-long gas cell filled with low-density krypton gas. It is noteworthy that, although our gas cell is only 10-mm-long, there are two holes drilled by the driver laser pulses at the two ends of the cell through which leakage of krypton gas inevitably occurs. The diameter of the holes was measured to be $\sim 2 \mathrm{~mm}$. As we will show below, the leaking gas from the holes could form a favorable density distribution of the krypton gas outside the gas cell for inducing filamentation and self-compression of the driver pulses, facilitating generation of IAPs. In addition, we find that control of the gas pressure, the driver pulse energy, and the cell length is crucial in this experiment. With these optimizations, we obtain single-shot spectra of intense EUV supercontinua covering the spectral range from 35 to $50 \mathrm{eV}$.

\section{EXPERIMENT}

The experimental setup is sketched in Fig. 1, which is exactly the same one used in our previous HHG experiments without any modification $[7,8]$. Although the previous experiments were focused on the HHG driven by long wavelengths, in this experiment, an 800-nm source was directly employed for HHG to utilize its full pulse energy. The laser system (Legend Elite Cryo PA, Coherent, Inc.) delivers 35-fs (FWHM) pulses with a central wavelength at $800 \mathrm{~nm}$ and a single-pulse energy of $\sim 11 \mathrm{~mJ}$. The beam diameter was measured to be $\sim 8.8 \mathrm{~mm}\left(1 / e^{2}\right)$. To generate Fourier-transform-limited driver pulses with shortest durations, we adjusted the distance between the grating pairs in the compressor of the femtosecond laser amplifier. A 10-mm-long gas cell filled with krypton gas was mounted in the vacuum chamber for generating high-order harmonics. The gas pressure can be adjusted in the range from 0 to 200 mbar for optimization of the HHG process. The backpressure inside the vacuum chamber was $\sim 10^{-3}$ mbar. A flat-field grating spectrometer equipped with a 


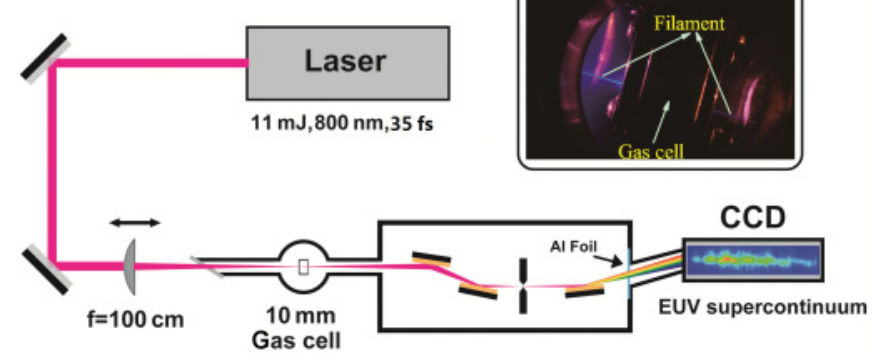

FIG. 1. (Color online) Schematic of the experimental setup. The same single filament can be seen at both sides of the gas cell as shown in the inset.

soft-x-ray CCD (Princeton Instruments, $1340 \times 400$ imaging array PI:SX 400) was used to characterize the harmonic emission. We employed a 150 -nm-thick aluminum foil to block the lower-order harmonics and the residual infrared pulses. Since the driver pulses were not CEP stabilized, all the harmonic spectra presented here were captured in single shots. Although our previous experiments were mostly carried out by focusing $\sim 1$-to-2-mJ, $\sim 35$-fs pulses with lenses of focal lengths ranging from 15 to $40 \mathrm{~cm}$, in this experiment, a significantly higher pulse energy of $\sim 11 \mathrm{~mJ}$ (i.e., the maximum pulse energy affordable by our amplifier) was used, and a loose focusing scheme with a 100-cm-focal length was employed. Under these conditions, we observed that filamentation occurred at gas pressures above $\sim 30$ mbar as indicated by the arrows in the inset of Fig. 1. A long filament channel can be clearly seen at both sides of the gas cell.

\section{RESULTS}

Figure 2(a) shows a typical two-dimensional (2D) singleshot HHG spectrum observed by focusing the Fouriertransform-limited $\sim 35$-fs, 11 -mJ pulses into the 10-mm-long gas cell filled with $\sim 30$-mbar krypton gas. Dramatically, we observed a supercontinuum covering the spectral range from $\sim 35$ to $\sim 50 \mathrm{eV}$ (signals below $\sim 35 \mathrm{eV}$ were not recorded due to the limited size of $x$-ray CCD). Figure 2(b) presents the EUV supercontinuum spectrum for the signal distributed on the black dashed line in Fig. 2(a) (i.e., the divergence angle of $0 \mathrm{mrad}$ ). By assuming that the phase of the EUV supercontinuum in Fig. 2(b) is flat, an 271-as IAP with a clean temporal profile can be generated as shown in Fig. 2(c). The intensity of satellite pulses is well below $3 \%$ of that of the main pulse.

We found that the generation of the EUV supercontinuum is sensitive to the CEP value of the driver pulses. Figures 3(a)3(c) present HHG spectra recorded in other single shots which preserve all the same experimental conditions as used for the generation of the supercontinuum spectrum in Fig. 2(a) except the CEP values as the CEP value of our femtosecond laser amplifier was not stabilized. The HHG spectra in Figs. 3(b) and 3(c) show strong periodic modulation, indicating that control of the CEP is still necessary in the generation of IAPs with this approach. Nevertheless, CEP-stabilized amplifiers with pulse energies up to tens of millijoules are already commercially available and are used worldwide for HHG
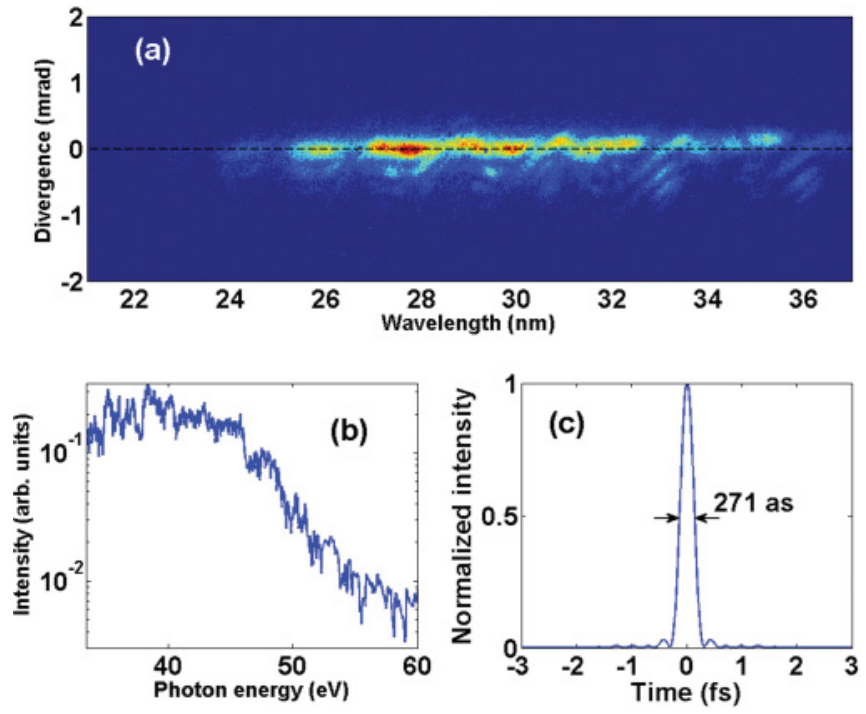

FIG. 2. (Color online) (a) A 2D single-shot supercontinuum spectrum generated by the Fourier-transform-limited $\sim 35$-fs, 11-mJ laser pulse, (b) EUV supercontinuum spectrum for the signal distributed on the black dashed line in Fig. 2(a), and (c) temporal profile of the single attosecond pulse by inverse Fourier transform of the spectrum in (b) provided that the attochirp is fully compensated.

experiments, making it possible for energy scaling up in single attosecond pulse generation using this technique. The driving pulse energy is also critical for the generation of the supercontinuum. Figure 3(d) shows a HHG spectrum recorded at a lower input energy of $8 \mathrm{~mJ}$. We can clearly see that the supercontinuum disappears, probably because of the weakened nonlinear effects caused by the lower pulse energy. Moreover, the HHG spectrum is also sensitive to the length of the gas cell. In Fig. 3(e), it can be seen that, when shorter gas cells (e.g., 2-mm long) are used, only discrete harmonics can be observed. This clearly indicates the important role of the propagation effects.

\section{DISCUSSION}

It is well known that the generation of IAPs requires the creation of a narrow time window within which the well-known HHG process occurs only once [1,2]. Thus, from a single-atom point of view, it should be impossible to produce the EUV supercontinuum with the multicycle $\sim 35$-fs driver pulses as shown in Fig. 2(a). However, HHG is also a highly nonlinear process extremely sensitive to propagation effects. As our driver pulses are very intense, filamentation can even occur at a low gas pressure of only $\sim 30$ mbar as evidenced in the inset of Fig. 1, leading to the spatiotemporal change in the driver pulses during the course of the HHG. Figure 4(a) shows that the spectrum of the driver pulses coming out from the HHG chamber is significantly broader than that of the input pulses. Moreover, the raw second-harmonic generation frequencyresolved optical gating (FROG) trace of the outgoing driver pulses is shown in Fig. 4(b), and the retrieved pulses are displayed in Fig. 4(d). Figure 4(d) shows that the driver pulses are split into several intensity spikes. We would like to stress that the retrieved pulse shown in Fig. 4(d) represents the temporal profile of the pulse exiting from the end of the 

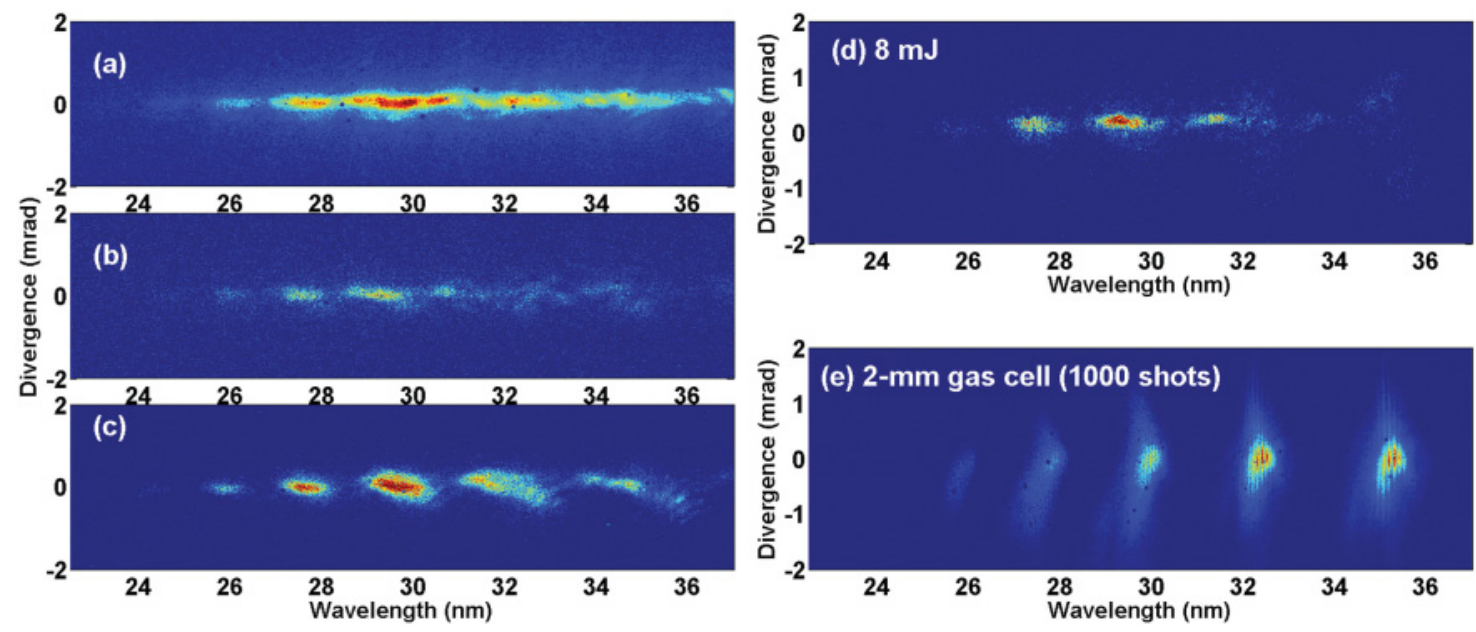

FIG. 3. (Color online) (a)-(c) HHG spectra generated in single shots with different CEP values, giving clear evidence on the CEP effect. (d) HHG spectrum generated at a lower energy of $8 \mathrm{~mJ}$. (e) HHG spectrum generated with a 2-mm-long gas cell.

filament, which is not necessarily the same as the driver pulse responsible for the generation of the EUV supercontinuum. For example, the driver pulse may only exist in the middle portion of the filament. However, the strong modulation of the driver pulses [Fig. 4(d)] provides clear evidence that they have undergone a dramatic spectral or temporal change during the propagation through the gas cell. Thus, there is no doubt that the observed EUV supercontinuum must, at least partially, be generated due to some propagation-induced effects, such as phase matching and/or ionization gating $[9,10]$. Particularly, it should be noticed that, in this high-energy regime, the driver pulses themselves can undergo dramatic changes during the propagation because of strong plasma generation. Therefore, for fully clarifying the underlying mechanism, sophisticated
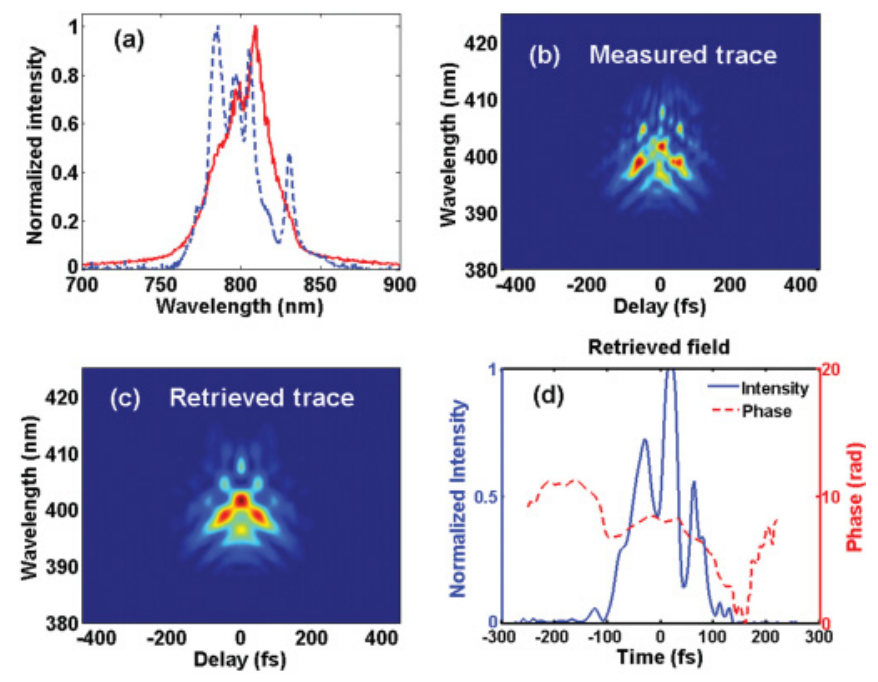

FIG. 4. (Color online) (a) Spectra of the original driver pulses (solid line) and the driver pulses coming out from the HHG chamber (dashed line). (b) Measured and (c) retrieved FROG traces of the driver pulses coming out from the gas cell. (d) Retrieved temporal intensity (solid line) and phase (dashed line) of the driver pulses. three-dimensional simulation tools, including the propagation effects, are needed $[11,12]$, which will be our future direction.

Besides being easy to operate, the use of the long gas cell, with two holes drilled at its two ends by the driver laser pulses, has several other advantages for both filamentation and HHG. First of all, unlike a gas jet which usually forms a decreasing gas density in the direction perpendicular to the propagation direction of the driver laser, the gas cell with holes at its two ends can form an axially symmetrical density distribution for the driver pulses. In addition, since the holes are drilled by the driver pulses, perfect alignment can automatically be achieved. Second, the spread of the gas by leaking out from the holes naturally forms a favorable density gradient for single filamentation at high pulse energies [13], which is very useful for scaling up the HHG yield by use of high-energy driver pulses. Third, both the length of the gas cell and the gas pressure can be easily tuned, which facilitates optimization of both propagation of the driver pulse and phase matching of the HHG.

\section{CONCLUSION}

In conclusion, we have successfully demonstrated generation of an EUV supercontinuum with Fourier-transformlimited $\sim 35$-fs, $11-\mathrm{mJ}$ driver pulses directly from an amplifier. Our experiment is carried out in a low-pressure noble gas with a conventional HHG experimental setup, which could lead to great simplification of the technology for attosecond pulse generation. With higher pulse energies, this technique also holds promise for the generation of intense IAPs using longerfocal-length lenses. Both these advantages will have important implementations for attosecond science and technology.

\section{ACKNOWLEDGMENTS}

This work was supported by the National Basic Research Program of China (Grant No. 2011CB808102) and the NSFC (Grants No. 11134010, No. 10974213, and No. 60825406). 
[1] F. Krausz and M. Ivanov, Rev. Mod. Phys. 81, 163 (2009).

[2] P. B. Corkum, Phys. Rev. Lett. 71, 1994 (1993).

[3] E. Goulielmakis et al., Science 320, 1614 (2008).

[4] G. Sansone et al., Science 314, 443 (2006).

[5] H. Mashiko, S. Gilbertson, C. Li, S. D. Khan, M. M. Shakya, E. Moon, and Z. Chang, Phys. Rev. Lett. 100, 103906 (2008).

[6] D. S. Steingrube, E. Schulz, T. Binhammer, M. B. Gaarde, A. Couairon, U. Morgner, and M. Kovacev, New J. Phys. 13, 043022 (2011).

[7] H. Xiong, H. Xu, Y. Fu, J. Yao, B. Zeng, W. Chu, Y. Cheng, Z. Xu, E. J. Takahashi, and K. Midorikawa, Opt. Lett. 34, 1747 (2009).
[8] H. Xu, H. Xiong, Z. N. Zeng, Y. X. Fu, J. P. Yao, R. X. Li, Y. Cheng, and Z. Z. Xu, Phys. Rev. A 78, 033841 (2008).

[9] Z. Chang, A. Rundquist, H. Wang, I. Christov, H. C. Kapteyn, and M. M. Murnane, Phys. Rev. A 58, R30 (1998).

[10] T. Pfeifer, A. Jullien, M. J. Abel, P. M. Nagel, L. Gallmann, D. M. Neumark, and S. R. Leone, Opt. Express 15, 17120 (2007).

[11] V. Tosa, H. T. Kim, I. J. Kim, and C. H. Nam, Phys. Rev. A 71, 063807 (2005).

[12] A. Couairon, H. S. Chakraborty, and M. B. Gaarde, Phys. Rev. A 77, 053814 (2008).

[13] A. Suda, M. Hatayama, K. Nagasaka, and K. Midorikawa, Appl. Phys. Lett. 86, 111116 (2005). 